\title{
Diagnosis and Management of Blunt Pancreatic Trauma
}

\author{
Dániel Kollár ${ }^{1}$, Tamás F. Molnár ${ }^{1,2}$, Péter Zsoldos ${ }^{1}$, Zoltán Benedek-Tóth ${ }^{1}$, Attila Oláh ${ }^{1}$
}

'Department of Surgery, Petz Aladár Teaching Hospital, Győr, Hungary

2Department of Operational Medicine, University Medical School of Pécs, Hungary

\section{ABSTRACT}

Introduction: Blunt or penetrating pancreatic trauma represents only $0.2-2 \%$ of all trauma cases and approximately $3-12 \%$ of all abdominal injuries. While treatment protocol debates of other intra-abdominal and thoracic organ injuries seem to reach comforting conclusions, satisfying evidence-based recommendations regarding the pancreas have not been released yet. However, high grade trauma of the pancreas can lead to substantial morbidity and mortality. The question is, when and how to treat it conservatively or operatively.

Objectives/Methods: This study is a review of contemporary literature on children and adult pancreatic trauma management strategies and findings. The purpose is to evaluate current classifications and the efficacy of subsequent non-operative and operative treatments. We list the established grading systems starting from physical examination, imaging diagnosis, to indications for surgery or conservative management, followed by post-treatment morbidity and mortality rates.

Conclusions: Current operative or non-operative management strategies are not based on randomized - or even, in fact, on prospective - clinical trials. Most of the available publications demonstrate small retrospective patient cohorts and expert opinions. To date, no convincing high level (at least Level III) evidence-based recommendations have been published in terms of treatment of these injuries. There is a general agreement, that the injury of the main pancreatic duct is the thin red line, dividing conservative and operative strategies. Low grade pancreatic injury can be treated conservatively not significantly different from protocols developed for mild pancreatitis of other origin. Pancreatic duct damage in adults requires either minimal invasive intervention or exploration and reconstruction/resection via laparotomy. Treatment strategies of high grade paediatric pancreatic injuries remain controversial. Associated organ injuries can mask the symptoms of pancreatic trauma. Missed main pancreatic duct injuries pose a clinically challenging situation with serious complications and considerable mortality. Pancreatic injury in polytrauma poses the highest risk. Present perspectives for survival of pancreatic injury as mono trauma varies between $95-100 \%$, while as a part of polytrauma, the mortality rate is as high as $30-35 \%$. Multicentre prospective, randomized clinical trials would be ideal to support optimal decision making. Heterogeneity of cases and relative rarity of the pathology makes the creation of such a database highly unlikely.

Key words: blunt abdominal trauma, pancreatic transsection, distal pancreatectomy, pseudocyst, interventional radiology

\section{INTRODUCTION}

Pancreatic injury makes up a relatively small proportion of trauma cases: the pancreas is affected in $0.2-2 \%$ of all trauma patients and in $2-12 \%$ of all

\author{
Corresponding author: \\ Attila Oláh, MD \\ Department of Surgery \\ Petz Aladár Teaching Hospital \\ 9024 Győr, Vasvári Pál utca 2-4 \\ Hungary \\ E-mail:drolaha@gmail.com)
}


abdominal injuries (1-7). Given its retroperitoneal location and comparatively protected milieu, an excessive force of blunt or penetrating trauma is required to damage the parenchyma of the pancreatic gland. This results in high rates (up to $82-85 \%$ ) of associated abdominal injuries $(4,8)$. While retrospective analyses report different origins of trauma in various age groups and areas around the world, they all agree on some key features: pancreas injury afflicts twice as many males as females, it presents with vague symptoms on physical examination and on radiology images. A missed diagnosis of significant pancreatic injury poses a very high risk of early and late complications in itself, while the associated injuries are responsible for the substantial mortality rates.

Reports from paediatric trauma centres are controversial in regards of management of child pancreatic injuries. Some institutions prefer early surgical resection of the damaged parts of the pancreatic parenchyma with quick recovery and early hospital discharge (9). However, there certainly is a trend towards conservative or minimal invasive management of even high grade injuries to avoid secondary morbidity related to the laparotomy itself (10-13). The classification of injuries, diagnostic findings and complications is the foundation of creating a statistically sound comparison of the efficacy of different treatment strategies (14). Since pancreatic injuries are rare and their treatment depends to a large extent on local institutional preferences, diagnostic capabilities and surgical expertise, high level evidences the management of blunt pancreatic trauma has not yet emerged. This study aims to review recent individual reports on pancreatic trauma to allow a deeper insight in the complexity of this potentially lethal injury.

\section{MECHANISM OF PANCREATIC INJURY}

The pancreas is surrounded by a robust musculoskeletal and parenchymal apparatus, which renders it relatively protected. This explains why only a low percentage of abdominal trauma is associated with pancreatic injury, and also the reason why any force capable of damaging the pancreas will cause collateral damage as well (i.e. liver, splenic, renal and/or vascular injury). Penetrating trauma (gunshot, stab wound) appears to be the main cause of pancreatic trauma in the United States and South Africa $(15,16,17)$, while in the rest of the world pancreatic trauma is mainly blunt and related to road traffic accidents (up to $54-85 \%$ ), sport injuries and falls $(4,18)$. Blunt abdominal trauma by bicycle handle bar is responsible for every third- second paediatric pancreatic trauma $(19,20)$. Blunt pancreatic trauma is a result of crushing force or compression of the pancreatic gland against the vertebral column causing either contusion, laceration or fracture of the parenchyma. The most common predilection point for injury is at the transition between the head and body (i.e. the neck) of the pancreas (21). The pancreatic body and neck are damaged by direct compression against the vertebral column, while the less common pancreas head or tail injuries develop due to blows to the flanks (22).

\section{PRESENTATION AND DIAGNOSIS}

The contained environment of the pancreas is equally beneficial and disadvantageous: minor lacerations, extravasations are self-limiting and are unlikely to cause diffuse peritoneal reactions. However, this aspect also mitigates clinical symptoms. The onset of abdominal symptoms in isolated pancreatic injury can be delayed by hours or even days after trauma (23). Isolated blunt pancreatic trauma $(11-17 \%$ of all pancreatic injuries) $(4,8)$ is easily missed in the early posttrauma phase, since it comes with non-specific vague symptoms: patients present with mild to severe epigastric pain and corresponding tenderness, nausea, vomiting and absent bowel sounds. Moreover, multiorgan injuries can mask the initial symptoms and divert the attention of the physician, especially in a hemodynamically unstable polytrauma setting.

Laboratory results such as leucocytosis and anaemia are alarming but not specific. Elevated serum amylase and lipase levels can raise suspicion of pancreatic injury and warrant further investigations but their sensitivity and specificity are only around $70-77 \%(4,5,22,24)$. A rising tendency in follow-up amylase levels during observation is much more of a help, but the actual levels do not predict the extent of organ injury $(2,19,23)$. Pseudocyst formation can be accompanied with persistent serum amylase elevation which can normalize once the pseudocyst has been treated or resolved. Serum lipase is useful to exclude salivary amylase elevation in extensive maxillofacial injuries (7).

Ultrasound (US) has low accuracy in diagnosing pancreatic injury, however it is widely used in the trauma setting (FAST scan: focused assessment with sonography for trauma) to identify free fluid or fluid collections indicating parenchymal and vascular injuries and thus the need for urgent laparotomy (25). Ultrasound is used for follow-up and for percutaneous drainage guidance of late pancreatic complications, such as pseudocysts and abscesses. Certain fluid 
collections can safely be targeted using endoscopic ultrasound (EUS). Contrast-enhanced ultrasound (CEUS) is an improved modality that needs further evaluation (3).

Computed tomography (CT) is generally accepted to be the gold standard in imaging provided that the patient is hemodynamically stable (26). CT results can be misleading, especially in the first 24 hours. When in doubt, it can be repeated to detect developing peri-pancreatic oedema, gland transection or fluid collections as the inflammation and secondary soft tissue changes evolve (5). To achieve maximum accuracy, the use of both oral and intravenous contrast enhanced helical CT is advised (slices' width $<5-7 \mathrm{~mm}$ ) (22). Still, this method cannot identify all pancreatic lesions correctly, as sensitivity and specificity are around $71-91 \%(20,27,28)$. Early, subtle signs of pancreatic injury are easy to miss, especially if only a small part of the parenchyma is damaged. Signs on CT suggesting significant pancreatic injury are: low attenuation line, free fluid, fluid collections (lesser sac, pararenal, peripancreatic, subphrenic), pleural effusion, inhomogeneous, edematous pancreas, separation of fragments, non-enhancement and atrophy of distal pancreas. There is no correlation between the number of CT signs and the severity of pancreatic injury. No single finding on CT scan is diagnostic, but the combination of pancreatic abnormalities can be suggestive of parenchymal and/or main pancreatic duct (MPD) injury, which is the key to adequate further management (22). CT can also be used for follow-up and for guiding percutaneous drainage once delayed complications have occurred.

Endoscopic retrograde cholangiopancreatography (ERCP) is considered to be the most accurate method in diagnosing pancreatic duct injury providing real-time images of the pancreatic duct and eventual extravasation or cut-off of the contrast substance (29). ERCP can reveal duct injuries that were obscure on initial CT scans. It is also useful in evaluating the cause of continuous high-output pancreatic fistulas and pseudocyst communication with pancreatic ducts. Complete MPD transection or 'disconnected duct syndrome' warrants surgical intervention. In select cases, ERCP can be therapeutic: partial or complete MPD injuries can be stented or bridged, transpapillary drainage can be obtained by sphincterotomy and pseudocysts can be drained either via the stomach or the duodenum. In small retrospective cohorts, endoscopic intervention was able to reduce the need and/or extent of surgical intervention by up to $42-90 \%$. Due to its invasive nature, it can be associated with pancreatitis and risk of infection. Post-ERCP pancreatitis rate is reported between $3 \%$ to $14 \%$ and in most cases it is mild and resolves with conservative management only (21). If stent migration, duct stricture or bleeding occur, they usually require repeat endoscopies. ERCP might not be available in every trauma centre and it does indeed require expertise, especially in the setting of polytrauma and in children. Apart from that, failed cannulation of the papilla of Vater or inadequate pancreatography can occur in up to $10 \%$ of patients (25).

Magnetic resonance cholangiopancreatography (MRCP) is considered to be a safe, non-invasive modality. Non-dynamic visualization is common therefore extravasation from a pancreatic duct injury might be missed (25). MRCP can also lead to misdiagnosis in the early phase (30). Accuracy can be improved with dynamic secretin-enhanced sequence (25). The major advantage of MRCP is that it avoids ERCP related complications and can visualize the parenchyma upstream of a complete pancreatic duct disruption, which is a blind spot in ERCP. Lack of collateral radiation makes it suitable for pregnant women and children as well. On the other hand, it cannot be used for therapeutic procedures as a guiding tool.

\section{CLASSIFICATION OPTIONS IN PANCREATIC TRAUMA}

In order to duly specify individual treatment and prognosis, certain scoring and grading systems have been introduced. Correct classifications need to fulfil certain principles: they should identify and discriminate the mechanism, location and extent of damage, different grades should be linked with different complications, treatment strategies and outcomes. Also, scoring systems have to be simple, objective and reproductible in order to allow comparison between different studies (14). Starting from patient admission following trauma, complex scoring systems [e.g. Injury Severity Score - ISS, Abbreviated Injury Score (24), Revised Trauma Score (31)] and their combinations (Trauma Score - Injury Severity Score - TRISS) are available to estimate the outcome and probability of survival of individual cases (14). These global trauma scores do not focus on pancreas or even torso injuries, therefore their applicability in the present field is limited. However these severity indexes are helpful in patient selection. Deciding between primary resection and reconstruction of complex injuries versus damage control surgery followed by a stabilization process with delayed secondary laparotomies relies significantly on these prognostic scores. 
Pancreas specific damage can be graded using already devised classifications. Each of the grading systems established so far underline the importance of presence/lack of having a MPD injury or not, as subsequent treatment, morbidity and mortality depend largely on it. Nevertheless, Lucas et al did not acknowledge the importance of duct injury, therefore it has lost its importance (32).

The Organ Injury Scaling of the American Association for the Surgery of Trauma (AAST - OIS) is the most accepted classification. Its proposed grading is compatible with the injury classes of associated organs. [33).

- Grade I Minor contusion or laceration. No ductal injury;

- Grade II Major contusion or laceration. No ductal injury;

- Grade III Complete transection of distal pancreas or distal pancreatic parenchymal injury with injury to pancreatic duct;

- Grade IV Proximal pancreatic transection or injury involving proximal duct or the ampulla;

- Grade V Massive disruption involving the head of pancreas.

Takishima et al. introduced a classification system using ERCP results in a retrospective cohort of pancreatic injuries (34):

- Class 1 Normal ERCP finding;

- Class 2a Contrast leak from side branch but is contained within the pancreas parenchyma;

- Class 2b Contrast leak from side branch to retroperitoneal space;

- Class 3a Main pancreatic duct injury in pancreas body or tail;

- Class 3b Main pancreatic duct injury in pancreas head.

The Cape Town grading system is a modified version of the original Takishima classification separating incomplete and complete MPD disruptions (35).

- Grade 1 Normal pancreatic duct;

- Grade 2 Main pancreatic duct intact. Injury to a branch of the pancreatic duct:

A Contrast contained within the pancreatic parenchyma;

B Contrast leak beyond the pancreatic parenchyma into the retroperitoneal space.

- Grade 3 Injury to main pancreatic duct. Duct continuity present:

A Injury to main pancreatic duct in body or tail with duct continuity;

B Injury to main pancreatic duct in the head with duct continuity.
- Grade 4 Complete main pancreatic duct division with no duct continuity:

A Complete main pancreatic duct division in body or tail with no duct continuity;

B Complete main pancreatic duct division in the pancreatic head with no duct continuity;

C Complete main pancreatic duct division with a leak or pseudocyst after resection of distal/proximal damaged pancreas.

The Frey-Wardell classification is useful in evaluating complex pancreatico-duodenal injuries. It assesses pancreatic and duodenum injuries separately (each anatomical category contains 3 classes) and them in a combined type (I-IV) in order to predict prognosis (36). Most publications analyse duodenal and pancreatic injuries separately. However, these organs form a physiological and anatomical single entity and require combined resection/reconstruction, especially in cases of trauma of the head and neck of the pancreas (16).

\section{Pancreas}

- Class I (P1) Capsular damage, minor parenchymal damage;

- Class II (P2) Partial/complete duct transection in the body/tail;

- Class III (P3) Major duct injury involving the head of pancreas or the intrapancreatic common bile duct.

\section{Duodenum}

- Class I (D1) Contusion, haematoma or partial thickness injury;

- Class II (D2) Full thickness duodenal injury;

- Class III (D3) Full thickness injury with > 75\% circumference injury or injury to the extrahepatic common bile duct.

\section{Combined}

Type I P1D1, P2D1, D2P1

Type II D2P2

Type III D3P1-2, P3D1-2

Type IV D3P3

\section{MANAGEMENT OF ADULT PANCREATIC INJURIES}

\section{Damage control principles}

Pancreatic injury is frequently associated with 
trauma of other organs and in most cases it does not dominate the clinical symptoms. All trauma admissions are to be managed according to the Advanced Trauma Life Support (ATLS) protocols. Hemodynamically unstable patients with a suspicion of major abdominal vascular or organ injuries need to have an immediate surgical exploration. In case of damage control surgerywhich cannot last longer than 90-120 mins, reconstructive interventions are to be planned at a later stage, when the potentially lethal factors (haemorrhage, shock, peritonitis, acidosis, hypothermia, coagulopathy), are treated successfully and the patient is stabilized (16). Penetrating trauma (mostly gunshots) can cause significantly more associated organ injuries than blunt abdominal trauma. These patients need damage control surgery for bleeding control, hollow viscus closure and/or drainage followed by planned re-look surgeries in a hemodynamically stable state significantly more frequently. Pancreatic damage can be overlooked even during explorative laparotomies if the surgeon is limited to damage control only. Exploration of the entire pancreatic gland is mandatory during laparotomy for abdominal trauma (5). If the main pancreatic duct injury is not confirmed (OIS Grade I-II), external drainage is sufficient. In case of major parenchymal laceration with damaged distal MPD (OIS Grade III), a left pancreatectomy is indicated, ideally with spleen preservation (37). Pancreatic head injuries with no signs of devitalization can also be drained. Definitive destruction of the pancreatic head and/or duodenum (Frey-Wardell Type III-IV injuries) needs individual assessment and in most cases, it needs multiple stage surgeries (e.g. resection +/- feeding jejunostomy followed by pancreaticoduodenectomy (Whipple's procedure) (8).

\section{Pancreatic trauma in hemodynamically stable patients}

Whenever a pancreatic injury is suspected and there is enough time for extensive diagnostic work-up, a helical multi-slice contrast-enhanced CT should be part of the diagnostic algorithm. Those with a normal pancreas on CT should be clinically re-evaluated on a regular basis and be investigated repeatedly if pancreatic injury is still not ruled out. Treatment of grade I-II injuries is very similar to the conservative management of acute pancreatitis (observation, analgesia, ulcer prophylaxis, bowel rest, nasogastric drainage, total parenteral nutrition (TPN) gradually advancing to oral feeding) (1). If pancreatic injury is visible on $\mathrm{CT}$, then (dynamic, secretin enhanced) MRCP should be carried out. If this shows an intact MPD, then subsequent ERCP is not necessary, provided that the patient remains observed. If the clinical suspicion of pancreatic duct injury is high (even with normal CT and MRCP findings), then ERCP should also be performed to assess the integrity of the MPD. If pancreatic duct injury is revealed, transpapillary duct stenting is advised, especially if the disruption is not complete and can be bridged with a stent. In select cases, complete or inaccessible duct transections can also be treated with a minimal invasive approach (transpapillary drainage and/or transmural drainage of pseudocysts). EUS guidance can be used to access smaller, non-bulging pseudocysts. Laparotomy should be limited in this group of patients to cases of main pancreatic duct injury where the minimal invasive approach is not feasible (e.g. complete duct transection in the early phase) (38). The above model requires centralized care, where the treating team includes endoscopy experts, surgeons, critical care physicians, and interventional radiologists (25).

Due to the initially vague symptoms, pancreatic trauma is easily missed on early presentation and radiology imaging $(22-61 \%)(5,22,39)$. Numerous studies have reported that delayed diagnosis (even by 24 hours) of substantial pancreatic trauma and/or main pancreatic duct injury leads to a significant rise in of morbidity and mortality $(5,18,20,40,41)$. The leakage of pancreatic juice causes autodigestion and necrosis in the retroperitoneal space. Local and subsequently systemic inflammatory responses are activated. If these are not contained in the retroperitoneal bed, they consequently lead to peritonitis, sepsis, shock, multiorgan failure and death. Failure of initial non-operative management can follow the same clinical course (6). These cases represent extremely challenging situations for surgeons and intensive therapists as well.

\section{SPECIAL ASPECTS OF PAEDIATRIC PANCREATIC TRAUMA}

Certain differences are to be highlighted between adult and paediatric abdominal trauma cases. Pancreatic injury in childhood is uncommon. According to huge trauma databases, it is present in $0.47 \%$ of all paediatric trauma admissions, in $1.7 \%$ of all abdominal trauma, and MPD injury is present in only $0.3 \%$ of trauma patients $(7,42,43)$. Children have a flatter diaphragm, thinner abdominal wall, and higher costal margin. They are smaller so traumatic forces are more prone to affect larger body proportions causing multi-organ injuries. Additionally, due to the fact that their musculoskeletal system is still developing, they only have a limited 
amount of subcutaneous and intra-abdominal fatty tissue, protective muscle layers along with flexible bone structure (4). These characteristics result in an increased risk of sustaining pancreatic damage from blows to the abdomen when compared to same forces in adults, especially in children in the low region of weight centiles (20). Penetrating injuries are less frequent in children. Blunt trauma by bicycle handlebar is the most common (up to $28-53 \%)(9,10,19,20,21)$, followed by road traffic accidents (including pedestrian or seat belt injuries) and falls. The possibility of child abuse must always be investigated in case of blurry or suspicious trauma history (4).

The available diagnostic means and their reliability are basically the same as in adult trauma. In footnote, the small size of children is not a contraindication for ERCP in expert hands (21).

Studies agree that paediatric damage control surgery should be based on the same principles as in adults: life-threatening abdominal injuries require immediate laparotomy, and once the abdomen is open, the pancreas can be assessed and treated accordingly. Low grade injuries need drainage only to evacuate proteolytic enzymes, grade III injury warrants distal pancreatectomy, while the less common grade IV-V trauma requires drainage followed by conditional pancreaticoduodenectomy (41).

It is also generally accepted, that grade I-II injuries resolve with conservative management and the occasional complications (formation of a pseudocyst, abscess or pancreatic fistula) can be handled with percutaneous or endoscopic interventions $(20,22,23,44)$.

Treatment recommendations for MPD injuries (grade III-V) are highly controversial in hemodynamically stable children. A wide spectrum of opinions is available in the relevant literature and the subsequent debates can be confusing. Paediatric surgeons prefer either non-operative treatment or early surgical intervention (45). In 2014, a questionnaire assessing therapeutic strategies for grade III pancreatic injuries in various paediatric units in four different countries revealed that if the patient presented 6 hours post injury, 10 surgeons would do a laparotomy, 8 would treat non-operatively and 3 would carry out endoscopic duct stenting via ERCP. If the same child with grade III injury were to be assessed 6 days post injury by the same surgeons, 4 would operate, 13 would treat conservatively and 4 would choose ERCP (46).

Supporters of early surgery and resection are basing their preference on prompt eradication of the source of potential complications. Children undergoing surgery tend to develop less pseudocysts and fistulae and are able to tolerate oral feeding earlier. This results in a shorter time of nasogastric or naso-jejunal feeding tube dependence, which is poorly tolerated by children. Consequently, TPN can be suspended sooner, avoiding villus atrophy and central-venous-catheter-related sepsis (9). The fast recovery results in shorter hospitalization, and therefore less costs and a mitigated level of psychologic trauma for the child $(6,21,43,47)$.

On the other hand, severe complications and death are very rare in paediatric isolated pancreatic trauma cases. Although those who favour non-operative management acknowledge that it can lead to increased morbidity (mainly fluid collections), but maintain that all of these morbidities resolve with time or with minimal invasive approach (48). Rejecting the option of laparotomy is based on the remarkable post-traumatic recovery capacity of children (49). Multiple studies report complete resolution of grade III MPD injuries and even recanalization of the pancreatic duct in the nonoperative group $(23,45,50,51)$. Also, laparotomies themselves can cause substantial postoperative complications (e.g. scars, wound infections, bleeding, dehiscence, delayed bowel movements, bowel obstruction due to adhesions, systemic inflammation). In isolated pancreatic injury, laparotomies aim to avoid pancreatic complications. However, the most dreaded risks, such as pancreatic fistulas and pseudocysts, frequently occur post-operatively as well (52). This phenomenon promotes the use of less invasive measures such as observation, supportive symptomatic treatment or percutaneous drainage of fluid collections. Wales et al. even advise against the use of ERCP in their study, since it has substantial morbidity and mortality, and children were to be treated conservatively regardless of the exact ductal anatomy (28).

The small cohorts examined in individual studies and the resulting lack of multi-centre randomized prospective trials delay the birth of a well-funded consensus regarding the management of paediatric pancreatic trauma.

\section{MORBIDITY AND MORTALITY RELATED TO PANCREATIC INJURY}

The aggressive nature of the proteolytic enzymes present in the pancreatic juice is the basis of various complications and the high morbidity $(30-70 \%)$ related to pancreatic trauma $(5,35)$. While associated organ injuries are responsible for early morbidity and mortality, pancreas-related complications generally present after surgery, or more importantly, in case of unrecognised or delayed diagnosis of pancreatic duct injury. 


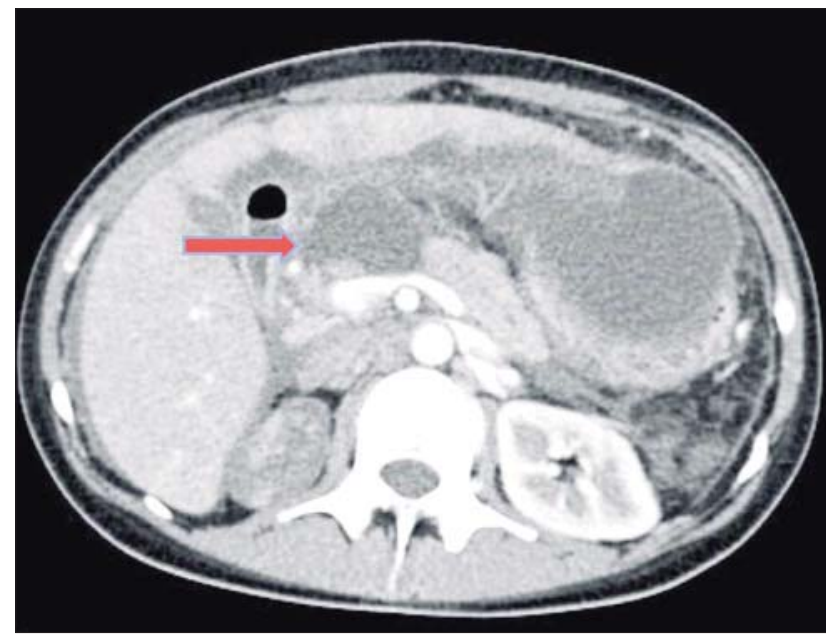

Figure 1 - CT image of a trauma - related complete pancreatic transection with pseudocyst formation (arrow), and surrounding free abdominal fluid suggesting cyst rupture in a 17-year-old girl

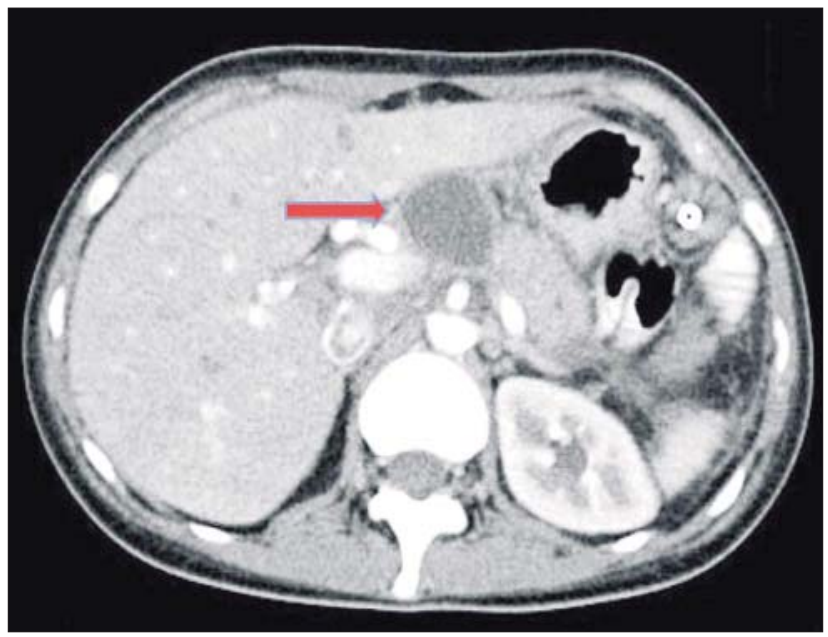

Figure 2 - Follow up CT image of the same child, 3 weeks later, after successful treatment with percutaneous drainage only. The free fluid resolved and the cyst has shrunk (arrow)
The most common complications are pancreatic fistulas and pseudocysts. They are caused by pancreatic juice leak and are mainly responsible for delayed hospital discharges and readmissions. An unrecognised MPD injury associated with postoperative leakage can also be the cause, typically requiring 2-4 weeks after trauma to fully develop. Bulging pseudocysts can cause gastric outlet obstruction, pain, and can eventually burst rupture, while fistulas present as intractable wound discharges. The spontaneous resolution of small pseudocysts is common, but the larger ones require active, sometimes repetitive treatment. More than $90 \%$ of traumatic pancreatic fistulas close within 8 weeks with conservative treatment (17). Fistulas which the grading of the depending on its output helps in determining prognosis (53). If the fistula is not solved by conservative management, endoscopic transpapillary drainage, stenting and/or sphincterotomy should be attempted. Pseudocysts can be managed by percutaneous external or preferably transmural internal drainage (pigtail or self-expanding stent introduced endoscopically via the stomach or the duodenum). Octreotide is widely used but it has not been proven to be beneficial in reducing complications. Despite all efforts, non-resolving pseudocysts and fistulas exist, requiring either cyst-gastrostomy or surgical resection of the part of the gland which communicates with these abnormalities.

Acute pancreatitis is mostly associated with grade I-II pancreatic injuries and with ERCP-related complications. It generally responds to non-operative treatment.

Intraabdominal abscess is a more severe complica- tion, which typically occurs if the fluid collection becomes infected. Its therapeutic approach is based on percutaneous external drainage and antibiotic therapy.

Pancreatopleural (transdiaphragmatic) fistula is a rarely reported sequale complication of blunt torso thoracic trauma.

Sepsis, peritonitis, shock and multi-organ failure are secondary processes originating from the generalized inflammatory response caused by the initial injury and subsequent infections. Late mortality is a consequence of these derailed mechanisms, which are unresponsive to comprehensive intensive care.

The mortality rates of pancreatic trauma vary widely between different reports (5-30\%)(5,54): isolated pancreatic injury is rarely lethal, especially in children (0-2\%). Complex damage to nearby organs or major retroperitoneal vessels constitutes the cause of shock and early mortality. Therefore, the death rate is highest in the damage control laparotomy group (45\%)(16). Sepsis and MOF due to initially unrecognised MPD injury leads to delayed mortality. Penetrating injuries have poorer prognosis, with a mortality of $5.1 \%$ for stab wounds and $21 \%$ for gunshots (16).

In order to render different studies comparable, classification is again useful to categorize various results. The Clavien-Dindo system is a promising attempt at organising future study interpretations regarding morbidity. It analyses cardiac, respiratory, neurologic, gastrointestinal and renal subgroups of postoperative impairments and grades complications from minor deviations to lethal consequences (Table 1) (55). 
Table 1 - Clavien-Dindo classification of surgical complications

Subgroups for Cardiac, Respiratory, Neurologic, Gastrointestinal and Renal complications.
Grade I Any deviation from the normal postoperative course without the need for pharmacological treatment or surgical, endoscopic, and
radiological interventions
Allowed therapeutic regimens are: drugs as antiemetics, antipyretics, analgetics, diuretics, electrolytes, and physiotherapy. This grade also
includes wound infections opened at the bedside
Grade II Requiring pharmacological treatment with drugs other than such allowed for grade I complications. Blood transfusions and total
parenteral nutrition are also included
Grade III Requiring surgical, endoscopic or radiological intervention
Grade IIla Intervention not under general anesthesia
Grade IIIb Intervention under general anesthesia
Grade IV Life-threatening complication requiring IC/ICU management
Grade IVa Single organ dysfunction (including dialysis)
Grade IVb Multiorgan dysfunction
Grade V Death of the patient

\section{DISCUSSION}

Blunt pancreatic trauma is uncommon and therefore a certain level of skepticism is useful upon interpretation of its literature. These studies have certain methodological limitations. All of the reports we cited present retrospective observational cohorts and only a few of them are multi-institutional. In a systematic review of the relevant literature, Haugaard et al. found no prospective/randomized clinical trials investigating treatment strategies (44).

Different populations can show unique characteristics of types of suffered trauma depending on wars, life style and the extent of motorization. The different strategy to establish the diagnosis (CT subtypes, use of ERCP/MRCP), the variable time from presentation to surgery, the diversity of associated injuries, the treatment preference of individual institutions and surgeons, and the prolonged time periods of each observation make analysis difficult and non-comparable (7). Morbidity and mortality rates reported so far have a surprisingly wide spectrum $(20-84 \%$ and $0-38 \%$, respectively) which is most probably caused by the various patient cohorts selected in different studies. Reports discussing all grades of isolated pancreatic injuries are more likely to have lower mortality rates (due to the good prognosis of the more common conservatively-managed low grade injuries). On the other hand, papers describing surgical cases only, with extensive multiorgan trauma, are associated with high mortality (16).

Increasingly sophisticated imaging and endoscopic techniques are essential in separating low-grade injuries from the high risk patient group. Minimally invasive methods, such as percutaneous or endoscopic transmural drainage stenting gain ground in substituting laparotomies across all age groups.

Recovery from pancreatic trauma depends on the presence of polytrauma, elapsed time from injury to diagnosis, and the extent of damage to the parenchyma, particularly to the main pancreatic duct (MPD). Low grade injuries are likely to resolve without operative intervention, but MPD transection is accompanied by severe morbidity if not treated in the early posttrauma phase. Lesions of the pancreatic duct can associate acute pancreatitis, pancreatic pseudocysts, fistulas, retroperitoneal abscesses which can lead to sepsis, multi-organ failure (MOF) and even death. Most authors agree on the importance surgical treatment has in such cases (distal pancreatectomy, pancreatico-jejunostomy, pancreaticoduodenectomy and/or drainage of fluid collections).

Figure 3 shows the suggested algorithm for treatment decisions. It can be regarded as a quintessence of evidence-based solutions open for further debate.

The pancreatic injuries of polytrauma patients are ideally treated in large trauma centres, where expertise and therapeutic means are readily available. A multidisciplinary involving trauma surgeons, pancreatic surgeons, intensive therapists, invasive radiologists and endoscopic experts should be readily available (6). The jury is still out on the question if an isolated trauma of the pancreas in a transportable, stable patient should ideally be treated in a specialised hepato-biliary centre or not. However, even considering this centralization of cases, most papers report a volume of c. 2-5 cases of significant pancreatic trauma per department per year. 


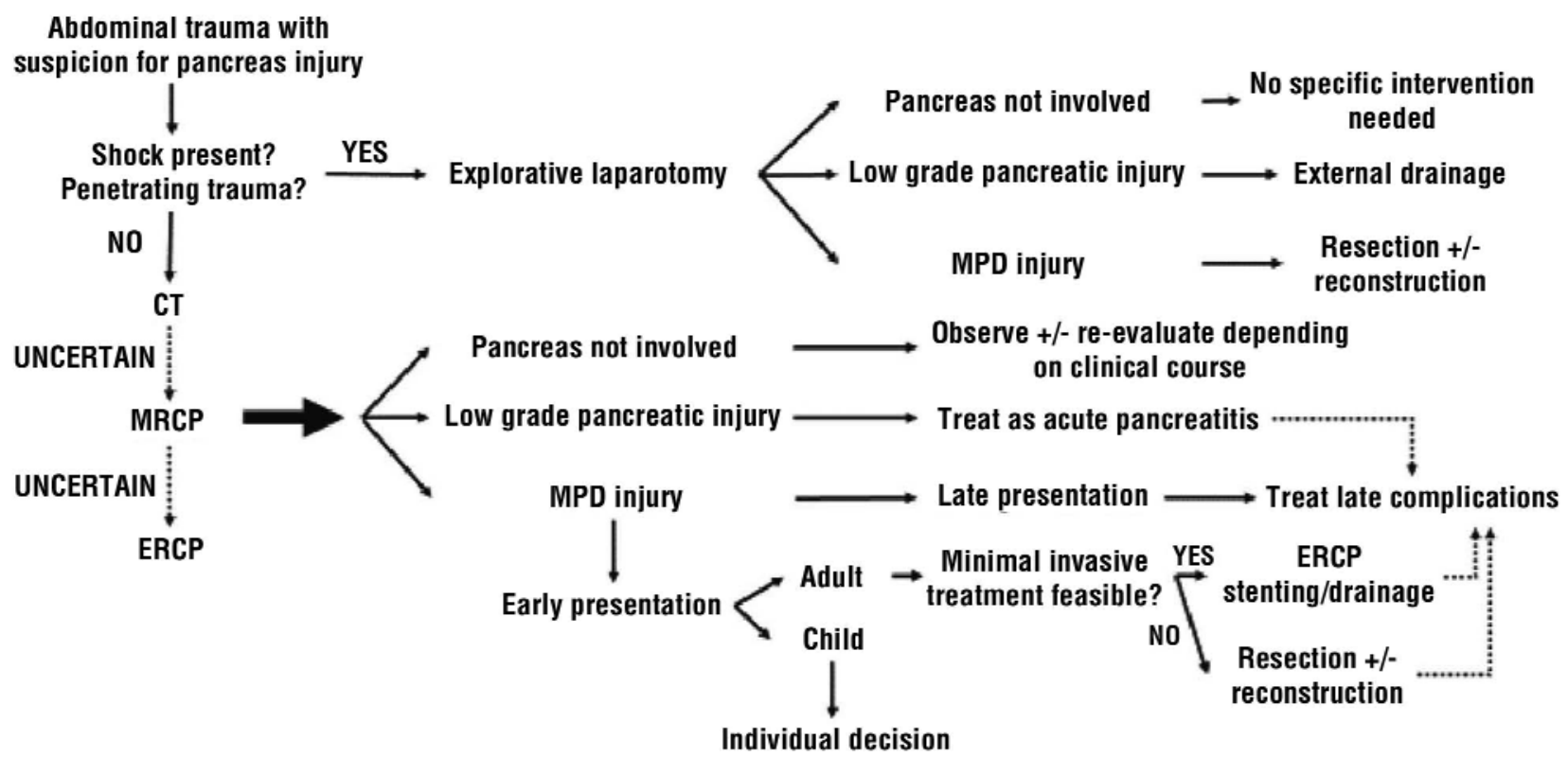

Figure 3 - Assessment of pancreatic trauma

This volume is insufficient for developing safe and sound protocols. On the other hand, due to the progression of treatment levels, we only see reports from the largest trauma centers, which is only the tip of the iceberg. A large proportion of less extensive pancreatic injuries are probably never published (or even recognised).

Generally-agreed classifications seem to be the key to obtain comparable objective data on etiology, diagnosis, treatment and outcomes of pancreatic injuries. This way we can exclude individual or institutional bias in order to reach higher levels of evidence concerning the correct management of these patients. The following data should be collected in the future: demographics, trauma score (ISS/RTS), mechanism of injury, presence of shock on admission, CT/MRCP/ERCP findings, location and grade of pancreatic injury, presence and extent of multi-organ injuries (Organ Injury Scaling), time from trauma to operation, type of surgical procedure (damage control/definitive surgery), number of transfusions, early $\beta 0$ day mortality, number of re-laparotomies, graded postoperative morbidity (Clavien-Dindo classification, pancreatic fistula grading using ISGPF guidelines), length of stay (8).

Despite the aforementioned methodological differences, certain consensuses have been developed over the past decades regarding pancreatic injuries. Trauma of the pancreatic gland is often accompanied by other organ injuries. The extent of these associated injuries will determine early survival rates while the less pronounced pancreatic injury affects late morbidity and mortality. Meticulous examination of the pancreas is essential in all patients undergoing damage control laparotomy in order to identify the extent of its injury. Hemodynamically stable patients with no imminent life-threatening conditions and with suspicion of pancreatic trauma should undergo extensive diagnostic work-up (blood samples, CT, MRCP +/- ERCP) to assess parenchymal status. The key to patient management is to identify/exclude the presence and location of main pancreatic duct injury. While complications from low grade parenchymal injuries will most likely resolve with conservative management, main pancreatic duct injuries in adults require surgical resection as early as possible. Management of grade III to V pancreatic blunt trauma in children is still under debate. There are advantages and disadvantages of both operative and conservative strategies. Late diagnosis of MPD disruption comes with increased morbidity and mortality in all age groups. Ductal lesions have to be diagnosed early to avoid pancreatic pseudocysts, fistulas and abscesses. Although minimally invasive methods (endoscopic and percutaneous) to treat duct injuries and subsequent complications have gained ground, availability of experienced surgical background is essential as a last resort. Failure of non-operative management is an indication for intraabdominal operative intervention (6). 


\section{CONCLUSIONS}

Pancreatic trauma remains a clinically challenging situation. The small number of cases, the variability of causes and the frequency of associated organ injuries lead to the lack of high level evidence based treatment recommendations. Early diagnosis is of paramount importance to avoid severe complications and mortality. However, none of the available diagnostic methods seem to be flawless. Involvement of the main pancreatic duct is the dividing line between conservative and operative management in adults, while reports on dealing with MPD injuries in children remains controversial. Minimally invasive methods are more and more widespread in early and delayed treatment as well, while complications not resolving with these techniques warrant the need for surgical resection. Multi-institutional, randomized prospective studies would be needed to correctly evaluate the superiority of either of these management strategies. Causative and demographic heterogeneity of patients and low case load per centres make the collection of a robust database highly unlikely.

\section{REFERENCES}

1. Potoka DA, Gaines BA, Leppäniemi A, Peizman AB. Management of blunt pancreatic trauma: what's new? Eur J Trauma Emerg Surg. 2015;41(3):239-50. doi: 10.1007/s00068-015-0510-3.

2. Debi U, Kaur R, Prasad KK, Sinha SK, Sinha A, Singh K. Pancreatic trauma: a concise review. World J Gastroenterol. 2013;19(47):900311.

3. Kumar A, Panda A, Gamanagatti S. Blunt pancreatic trauma: A persistent diagnostic conundrum? World J Radiol. 2016;8(2):15973. doi: 10.4329/wjr.v8.i2.159

4. Takishima T, Sugimoto K, Asari Y, Kikuno T, Hirata M, Kakita A, et al. Characteristics of pancreatic injury in children: a comparison with such injury in adults. J Pediatr Surg. 1996:31(7):896-900.

5. Girard E, Abba J, Arvieux C, Trilling B, Sage PY, Mougin N, et al Management of pancreatic trauma. J Visc Surg. 2016;153(4):25968. doi: 10.1016/j.jviscsurg.2016.02.006. Epub 2016 Mar 16.

6. Mattix KD, Tataria M, Holmes J, Kristoffersen K, Brown R, Groner J, et al. Pediatric pancreatic trauma: predictors of nonoperative management failure and associated outcomes. J Pediatr Surg. 2007:42(2): 340-4

7. Jobst MA, Canty TG, Lynch FP. Management of pancreatic injury in pediatric blunt abdominal trauma. J Pediatr Surg. 1999;34(5):81823; discussion 823-4.

8. Krige JEJ, Kotze UK, Nicol AJ, Navsaria PH. Isolated pancreatic injuries: An analysis of 49 consecutive patients treated at a Level 1 Trauma Centre. J Visc Surg. 2015:152(6):349-55. doi: 10.1016/j.jviscsurg.2015.09.010. Epub 2015 0ct 23.

9. Beres AL, Wales PW, Christison-Lagay ER, McClure ME, Fallat ME Brindle ME. Non-operative management of high-grade pancreatic trauma: Is it worth the wait? J Pediatr Surg 2013;(48):1060-4.

10. Paul MD, Mooney DP. The management of pancreatic injuries in children: operate or observe. J Pediatr Surg. 2011;46(6):1140-3. doi: 10.1016/j.jpedsurg.2011.03.041.

11. Coelho DE, Ardengh JC, Carbalo MT, de Lima-Filho ER, Baron TH Coelho JF. Clinicopathologic characteristics and endoscopic treatment of post-traumatic pancreatic pseudocysts. Pancreas.
2011;40(3):469-73. doi: 10.1097/MPA.0b013e31820bf898

12. Rogers SJ, Cello JP, Schecter WP. Endoscopic retrograde cholangiopancreatography in patients with pancreatic trauma. J Trauma. 2010; 68(3):538-44. doi: 10.1097/TA.0b013e3181b5db7a.

13. Bhasin DK, Rana SS, Rao C, Gupta R, Verma GR, Kang M, et al. Endoscopic management of pancreatic injury due to abdominal trauma. JOP. 2012;13(2):187-92.

14. Oniscu GC, Parks RW, Garden OJ. Classification of liver and pancreatic trauma. HPB (Oxford). 2006;8(1):4-9. doi: 10.1080/ 13651820500465881.

15. Wisner DH, Wold RL, Frey CF. Diagnosis and treatment of pancreatic injuries. An analysis of management principles. Arch Surg. 1990; 125(9):1109-13.

16. Krige JE, Kotze UK, Setshedi MS, Nicol AJ, Navsaria PH. Surgical management and outcomes of combined pancreaticoduodenal injuries: Analysis of 75 consecutive cases. J Am Coll Surg. 2016; 222(5):737-49. doi: 10.1016/j.jamcollsurg.2016.02.005

17. Krige JEJ, Kotze UK, Nicol AJ, Navsaria PH. Morbidity and mortality after distal pancreatectomy for trauma: A critical appraisal of 107 consecutive patients undergoing resection at a Level 1 Trauma Centre. Injury. 2014;45(9):1401-8. doi: 10.1016/j.injury.2014.04.024

18. Haulik L, Tóth B, Issekutz A, Gartner B. Pancreatic injury in blunt abdominal trauma: early versus late diagnosis and surgical management. Magy Seb. 2001;54(5):309-13. Hungarian

19. de Blaauw I, Winkelhorst JT, Rieu PN, van der Staak FH, Wijnen MH, Severijnen RSVM, et al. Pancreatic injury in children: good outcome of nonoperative treatment. J Pediatr Surg. 2008;43(9):1640-3. doi: 10.1016/j.jpedsurg.2008.03.061.

20. Houben CH, Ade-Ajayi N, Patel S, Kane P, Karani J, Devlin J, et al. Traumatic pancreatic duct injury in children: minimally invasive approach to management. J Pediatr Surg. 2007;42(4):629-35.

21. Rescorla FJ, Plumley DA, Sherman S, Scherer III LR, West KW, Grosfeld JL. The efficacy of early ERCP in pediatric pancreatic trauma. J Pediatr Surg 1995:30(2):336-40.

22. Shilyansky J, Sena LM, Kreller M, Chait P, Babyn PS, Filler RM, et al. Nonoperative management of pancreatic injuries in children. $J$ Pediatr Surg. 1998;33(2):343-9.

23. Maeda K1, Ono S, Baba K, Kawahara I. Management of blunt pancreatic trauma in children. Pediatr Surg Int. 2013;29(10):1019-22. doi: 10.1007/s00383-013-3402-9.

24. Duchesne JC, Schmieg R, Islam S, Olivier J, McSwain N. Selective nonoperative management of low-grade blunt pancreatic injury: are we there yet? J Trauma 2008:65(1):49-53.

25. Bhasin DK, Surinder S Rana SS, Rawal P. Endoscopic retrograde pancreatography in pancreatic trauma: need to break the mental barrier. J Gastroenterol Hepatol. 2009;24(5):720-8. doi: 10.1111/j. 1440-1746.2009.05809x. Epub 2009 Mar 12

26. Ruszinkó V, Willner P, Oláh A. Pancreatic injury from blunt abdominal trauma in childhood. Acta Chir Belg. 2005:105(3):283-6.

27. Garvey EM, Haakinson DJ, McOmber M, Notrica DM. Role of ERCP in pediatric blunt abdominal trauma: a case series at a level one pediatric trauma center. J Pediatr Surg. 2015;50(2):335-8. doi: 10.1016/j.jpedsurg.2014.08.017. Epub 2014 Dec 23.

28. Wales PW, Shuckett B, Kim PCW. long-term outcome after nonoperative management of complete traumatic pancreatic transection in children. J Pediatr Surg. 2001;36(5):823-7.

29. Snajdauf J, Rygl M, Kalousová J, Kucera A, Petrů O, Pýcha K, et al. Surgical management of major pancreatic injury in children. Eur $\mathrm{J}$ Pediatr Surg. 2007;17(5):317-21

30. Lin BC, Chen RJ, Fang JF, Hsu YP, Kao YC, Kao JL. Management of blunt major pancreatic injury. J Trauma. 2004;56(4):774-8.

31. Champion HR, Sacco WJ, Copes WS, Gann DS, Gennarelli TA, Flanagan ME. A revision of the Trauma Score. J Trauma 1989;29(5): 623-9.

32. Lucas CE. Diagnosis and treatment of pancreatic and duodenal injury. Surg Clin North Am. 1977;57(1):49-65.

33. Moore EE, Cogbill TH, Malangoni MA, Jurkovich GJ, Champion HR, Gennarelli TA, et al. Organ injury scaling. II. Pancreas, duodenum, small bowel, colon, and rectum. J Trauma. 1990;30(11):1427-9.

34. Takishima T, Hirata M, Kataoka Y, Asari Y, Sato K, Ohwada T, et al. 
Pancreatographic classification of pancreatic ductal injuries caused by blunt injury to the pancreas. J Trauma. 2000;48(4):745-51; discussion 751-2.

35. Krige JEJ, Kotze UK, Navsaria PH, Nicol AJ. Endoscopic and operative treatment of delayed complications after pancreatic trauma: An analysis of 27 civilians treated in an academic Level 1 Trauma Centre. Pancreatology. 2015;15(5):563-569. doi: 10.1016/ j.pan.2015.06.008. Epub 2015 Jul 8.

36. Frey CF, Wardell JW. Injuries to the pancreas. In: Trede M, Carter DC, editors. Surgery of the pancreas. Edinburgh: Churchill Livingstone; 1993. p. 565-89.

37. Malgras B, Douard R, Siauve N, Wind P. Management of left pancreatic trauma. Am Surg. 2011;77(1):1-9.

38. Biffl WL, Moore EE, Croce M, Davis JW, Coimbra R, Karmy-Jones R, et al. Western Trauma Association critical decisions in trauma management of pancreatic injuries. J Trauma Acute Care Surg. 2013; 75(6):941-6.

39. Snajdauf J, Rygl M, Kalousová J, Kucera A, Petrů 0, Pýcha K, et al. Surgical management of major pancreatic injury in children. Eur $\mathrm{J}$ Pediatr Surg. 2007 Oct; 17(5): 317-21.

40. Oláh A, Issekutz A, Haulik L, Makay R. Pancreatic transection from blunt abdominal trauma: early versus delayed diagnosis and surgical management. Dig Surg. 2003;20(5):408-14.

41. Clendenon JN, Meyers RL, Nance ML, Scaife ER. Management of duodenal injuries in children. J Pediatr Surg 2004;(39):964-8.

42. Sivit CJ, Eichelberger MR, Taylor GA, Bulas DI, Gotschall CS, Kushner DC. Blunt pancreatic trauma in children: CT diagnosis. AJR Am J Roentgenol. 1992;158(5):1097-100.

43. Meier DE, Coln DA, Hicks BA, Guzzetta PC. Early operation in children with pancreas transection. J Pediatr Surg. 2001; 36(2):341-4.

44. Haugaard MV, Wettergren A, Hillingsø JG, Gluud C, Penninga L. Non-operative versus operative treatment for blunt pancreatic trauma in children. Cochrane Database Syst Rev. 2014;(2):CD009746. doi: 10.1002/14651858.CD009746.pub2.
45. Gearman D, Casas-Melley AT. High-grade pancreatic trauma in pediatric patients: two cases of successful non-operative management. S D Med. 2014;67(12):499-503.

46. Westgarth-Taylor C, Loveland J. Paediatric pancreatic trauma: a review of the literature and results of a multicentre survey on patient management. S Afr Med J. 2014;104(11 Pt 2):803-7.

47. Iqbal CW, Peter SDS, Tsao K, Cullinane DC, Gourlay DM, Ponsky TA, et al. Operative vs nonoperative management for blunt pancreatic transection in children: multi-institutional outcomes. J Am Coll Surg. 2014;218(2):157-62.

48. Wood JH, Partrick DA, Bruny JL, Sauaia A, Moulton SL. operative vs nonoperative management of blunt pancreatic trauma in children. $J$ Pediatr Surg. 2010:45:401-6.

49. Kouchi K, Tanabe M, Yoshida H, Iwai J, Matsunaga T, Ohtsuka Y, et al. Nonoperative management of blunt pancreatic injury in childhood. J Pediatr Surg. 1999;34(11):1736-9.

50. Dixit P, Sharma V, Singh KR, Thapa BR, Rathore M. Conservative management of a case of traumatic pancreatitis in childhood: a case report. Eur Rev Med Pharmacol Sci. 2014;18(12):1687-9.

51. Arkovitz MS, Garcia VF. Spontaneous recanalization of the pancreatic duct: case report and review. J Trauma. 1996;40(6):1014-6.

52. Kollár D, Huszár T, Pohárnok Z, Cselovszky É, Oláh A. A review of techniques for closure of the pancreatic remnant following distal pancreatectomy. Dig Surg. 2016;33(4):320-8. doi: 10.1159/ 000445017. Epub 2016 May 25.

53. Bassi C, Dervenis C, Butturini G, Fingerhut A, Yeo C, Izbicki J, et al. Postoperative pancreatic fistula: an international study group (ISGPF) definition. Surgery. 2005;138(1):8-13.

54. Krige JE, Kotze UK, Setshedi M, Nicol, AJ, Navsaria PH. Prognostic factors, morbidity and mortality in pancreatic trauma: a critical appraisal of 432 consecutive patients treated at a Level 1 Trauma Centre. Injury. 2015;46(5):830-6. doi: 10.1016/j.injury.2015.01.032.

55. Dindo D, Demartines N, Clavien PA. Classification of surgical complications - a new proposal with evaluation in a cohort of 6336 patients and results of a survey. Ann Surg. 2004;240(2):205-13. 